\title{
Pelizaeus-Merzbacher disease in patients with molecularly confirmed diagnosis
}

\author{
Hanna Mierzewska ${ }^{1}$, Ewa Jamroz ${ }^{2}$, Tomasz Mazurczak ${ }^{1}$, Dorota Hoffman-Zacharska ${ }^{3}$, Elżbieta Szczepanik ${ }^{1}$ \\ ${ }^{1}$ Clinic of Child and Adolescence Neurology, Institute of Mother and Child, Warsaw, ${ }^{2}$ Department and Clinic of Paediatrics \\ and Neurology of Developmental Age, Upper Silesia Centre of Child's Health, Katowice, ${ }^{3}$ Department of Medical Genetics, \\ Institute of Mother and Child, Warsaw, Poland
}

\begin{abstract}
Pelizaeus-Merzbacher disease (PMD) is X-linked hypomyelinating leukodystrophy caused by mutations of the PLP1 gene, which codes the proteolipid protein 1. The result of mutations is abnormal myelination-hypomyelination and dysmyelination of cerebral white matter, and in some form of the disease hypomyelinating peripheral neuropathy. DNA samples from 68 patients suspected of PMD due to the clinical course and hypomyelination at magnetic resonance imaging (MRI) were analyzed. Medical history and detailed clinical course of PMD patients were also analyzed. Different mutations of the PLP1 gene were detected in 14 boys from 11 families ( 20\%). Amongst the molecularly confirmed patients, 13 presented classical PMD forms but clinical phenotypes varied in the severity even amongst siblings. One patient presented a severe connatal form. One mother, obligate carrier, presented complicated SPG2 (spastic paraparesis). There was no phenotype-genotype correlation in our material. In many cases PMD was suspected with a delay of many years, sometimes only after birth of another affected child in the family. Pelizaeus-Merzbacher disease was most frequently misdiagnosed as cerebral palsy.
\end{abstract}

Key words: Pelizaeus-Merzbacher disease, hypomyelination, dysmyelination, leukodystrophy, PLP1 gene mutations, MRI.

\section{Introduction}

Pelizaeus-Merzbacher disease (PMD) is X-linked recessive hypomyelinating leukodystrophy resulting from mutations in the PLP1 gene. The disease is allelic with spastic paraplegia type 2 (SPG2). The gene codes two protein isoforms, PLP1, the major component of myelin in the central nervous system and DM20 present in the peripheral nervous system $[2,8]$. Until now four phenotypes of PMD/SPG2 have been described. They include the severe inborn, classical,
PLP1-null (with peripheral neuropathy) form of PMD, and the uncomplicated or complicated SPG2 [1-3]. Neuropathological findings in PMD include widespread lack or reduction of myelin sheets, sometimes with patchy appearance of perivascular areas (tigroid pattern) and with relatively preserved neurons and their processes [2]. In our material various pathogenic PLP1 gene mutations were detected in 15 patients.

Thirteen cases have been classified as the classical form according to international clinical, electrophysiological and brain magnetic resonance imaging 
(MRI) criteria of hypomyelination. They manifested variable clinical course, which could be observed even in siblings. One patient presented with the severe connatal form. One mother who was the obligate carrier manifested signs and symptoms of a complicated form of SPG2. Diagnosis of PMD was established with a delay of several years, most frequently after delivery of another affected child in the family. Most frequently PMD was misdiagnosed as cerebral palsy (CP). Below, we present a clinical pattern of the disease in patients with molecularly confirmed PMD and analyze causes of the delay in the appropriate diagnosis.

\section{Material and methods}

The analyzed material included DNA samples from 68 boys and their 58 mothers, from different centers in Poland from 2007. The inclusion criteria were hypomyelination at MRI according to R. Schiffmann and van der Knaap, including a hyperintensity of the white matter in T2 and Flair [8], characteristic brain auditory evoked potentials (decreased or absent brainstem auditory evoked potentials of waves III-V) as well as the clinical pattern and medical history. Clinical PMD phenotypes were established according to international clinical criteria as classic (onset within the first 5 years of life with the nystagmus occurring within 1-2 months of age, initial hypotonia followed by spastic quadriparesis, ataxia, titubation, dystonia, athetosis, and cognitive decline), connatal (constant nystagmus, general hypotonia, preserved deep tendon reflexes microcephaly, severe developmental delay, marked pre- and postnatal growth retardation) and complicated SPG2 (spastic paraparesis, incontinence, dementia) $[1,2,8]$. Pedigree analyses of all families were conducted [4,5].

Analysis of DNA samples taken from peripheral blood was performed as described in the earlier paper [4]. At first, investigation for deletion/duplication was performed by MLPA technique and in cases without PLP1 duplications we looked for the presence of the point mutations [4].

\section{Results}

Diagnosis of PMD was established in 14 boys from 11 families. Ten mothers proved to be carriers, including one symptomatic carrier with signs of a complicated spastic paraparesis. The familial cases included two sets of siblings, each with two affected brothers, in one family two first cousins were affected. Respective clinical and molecular data are listed in Table I.

\section{Clinical data}

The classical form of PMD of different severity has been diagnosed in 13 patients. One patient manifested a severe connatal form. In patients with the classical PMD the first signs and symptoms were noted between the $1^{\text {st }}$ and the $8^{\text {th }}$ month of life. They included nystagmus, axial hypotonia and a delayed psychomotor development, particularly concerning motor skills. Gradually, with maturation of their nervous system they developed tremor of the head and/or oscillating movements of the head (titubation), linked to nystagmus. Permanent stridor was present in some patients; in most severely affected children it was evident only while crying. In the second half-a-year or the second year of life, cerebellar ataxia became obvious within the trunk and extremities, as well as dyskinesias (mainly choreoathetoid movements), overlapping with intentional movements. In one of the patients, cerebellar ataxia persisted till the $8^{\text {th }}$ year of life. Deep tendon reflexes were constantly present but elevated reflexes were noted most frequently after the $12^{\text {th }}$ month of life. Spasticity did not appear until the second up to fourth year of life; in older children it was more pronounced in lower extremities. Older children manifested mainly focal or multifocal dystonia overlapping with the pyramidal signs. All the thirteen patients had psychomotor retardation, but an emotional and mental development was relatively better preserved than motor activities. One of the patients manifested normal intelligence in Leiter International Performance Scale. Ophthalmological examination detected small, pale optic discs in all patients. Nevertheless, vision was relatively well preserved. A clear neurological deterioration and regress in the development were observed after a few years, and in one case, after more than ten years.

The patient with the connatal form was delivered with signs of intrauterine growth retardation. His psychomotor development was profoundly retarded and failure to thrive was evident. His clinical pattern involved a mild, constant nystagmus, extreme muscular axial and limbs hypotonia with preserved deep tendon reflexes and slight involuntary movements of hands and feet [5]. The mother affected of SPG2 had slowly progressing spasticity of lower limbs, urine incontinence and cognitive decline. Her brain MRI revealed abnormal diffuse hypomyelination/dysmyelination. 


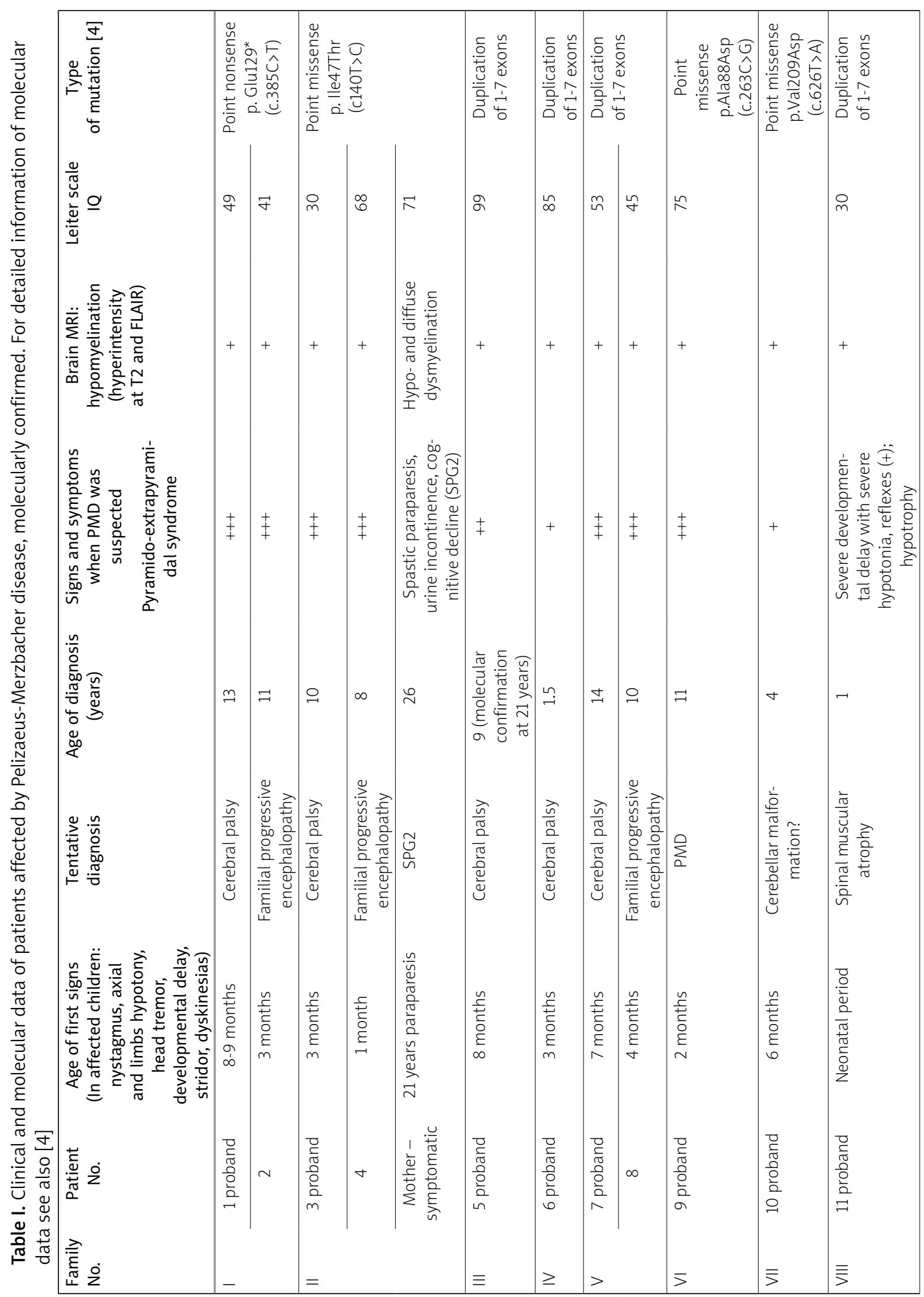




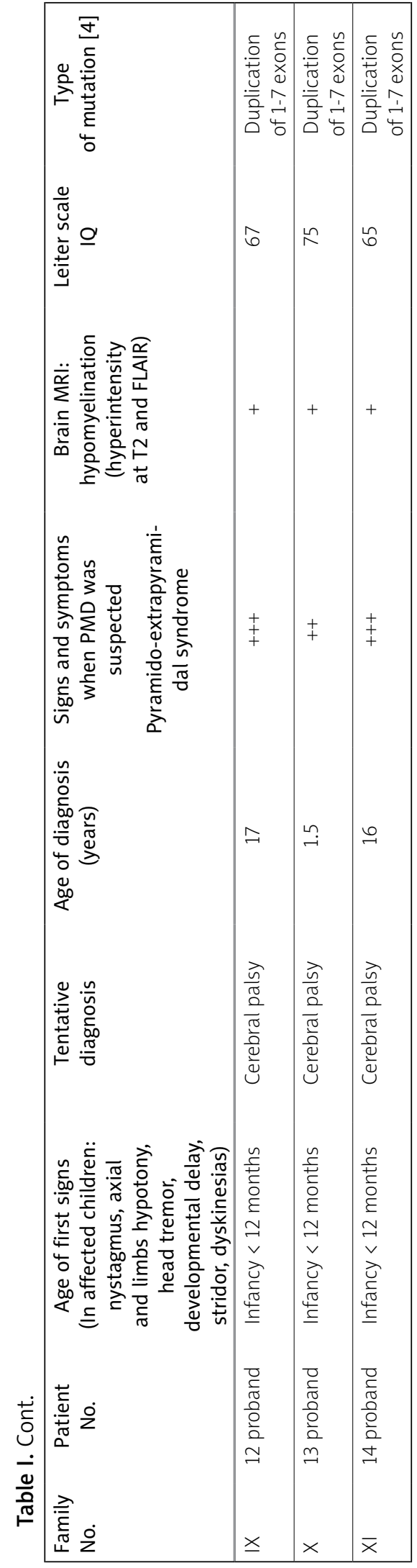

In 11 out of the 14 boys (family I, II, V - see Table I), PMD was preceded by diagnosis of cerebral palsy, including three patients diagnosed with its cerebellar form and one patient suspected of cerebellar malformation. All of them were probands (the first affected family members). Just only after a few up to more than ten years, when the disorders deteriorated, or after delivery of similarly affected second child, the patients were referred to geneticists as familial progressive encephalopathy. In 4 patients, PMD was diagnosed in infancy: three brothers of an affected patient and an 11-month-old proband consulted by an experienced physician. In all the cases the decision to perform molecular testing was taken following detection of hypomyelination on MRI imaging (Fig. 1).

\section{Molecular finding}

The molecular analysis demonstrated duplication of the entire gene but of a slightly different range in seven cases (family III, IV, VII, IX, X, XI), and four point mutations of missense (family I) and nonsense types (family II, V, VI) in exons 2, 3 and 4 (see Table I) [4].

\section{Discussion}

Pelizaeus-Merzbacher disease is the best known leukodystrophy with hypomyelination. In the Czech Republic and in Germany its incidence is estimated at $1: 90,000$ to $1: 100,000$ live births [2,8]. Thus, we may conclude that in Poland many cases of PMD/ SPG2 remain undiagnosed. The small group of 15 patients presented above, referred from various pediatric Polish centers since 2007 necessitates considering the reasons for such a low diagnostic efficacy. The first contributing factor may be unspecific character of clinical signs and symptoms. A similar clinical pattern can be found in several inborn and acquired diseases, of which the most frequently encountered is cerebral palsy. The "labelled" patient is referred to rehabilitation centers. In the early period of the disease the increasing muscular tonus is thought to reflect positive effects of rehabilitation. So the progress of the disease is falsely taken for improvement. Additionally some other disturbances alleviate or are modified by the maturation of the nervous system. This also makes the physicians blind to the possibility of another diagnosis. Further development of spasticity is thought to be related to evolution of cerebral palsy and the affected children probably may not return to diagnostic centers. 

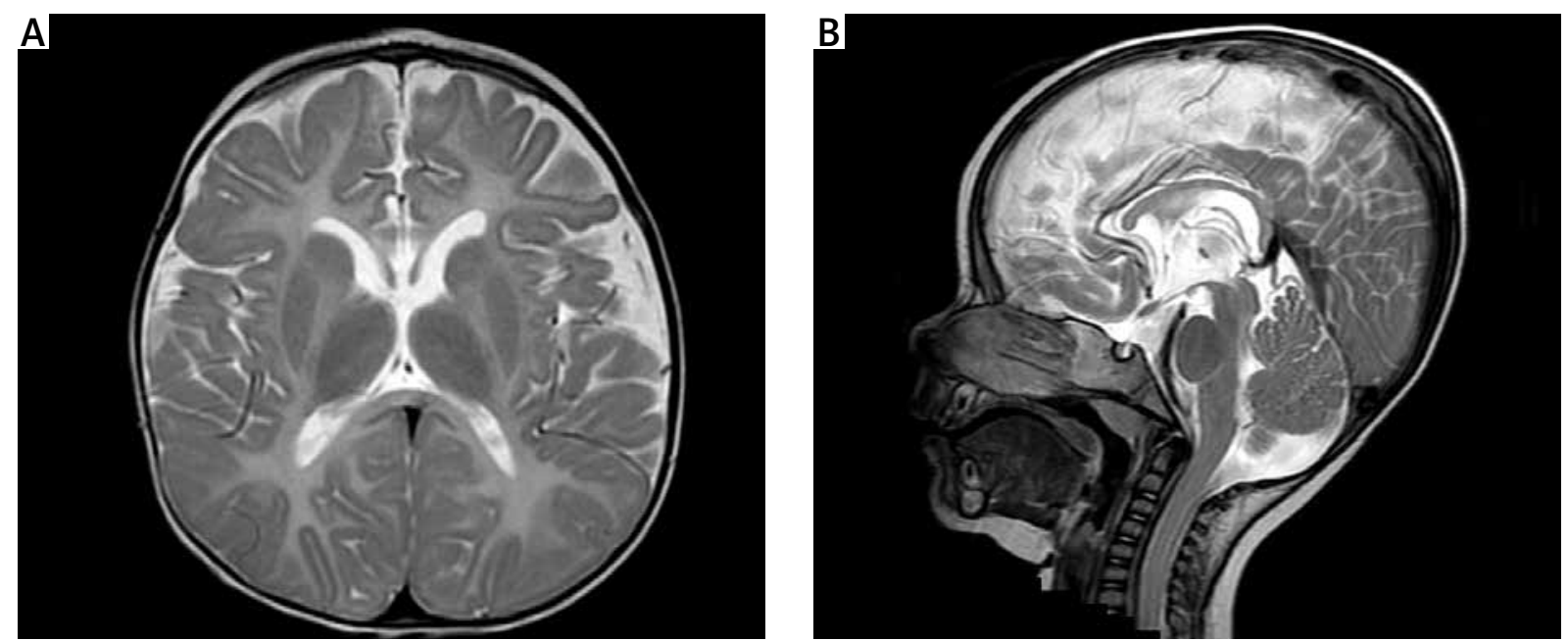

Fig. 1. A) T2-weighted magnetic resonance imaging of the brain of a 1.5-year-old patient affected by PelizaeusMerzbacher disease. Transverse section - mild hyperintensity (as compared to cortex) of whole white matter is visible, also in posterior limb of internal capsules. B) Sagittal T2-weighted magnetic resonance imaging - see very thin corpus callosum due to the decreased white matter volume.

A "red flag" does not appear until the day when another boy in the patient's family is delivered with similar signs and symptoms. As a result, the former diagnosis is revised and the child is referred to a geneticist.

The delays and errors in diagnosis of PMD are probably caused also by the currently prevalent view that all neurodegenerative diseases always manifest a gradual progressive deterioration. In contrast, the majority of children with PMD, despite neurological disturbances, show progress of various range in their psychomotor, emotional and intellectual development. They frequently acquire a number of abilities due to the enormous developmental potential of the young organism. Most of our patients started to demonstrate evident, gradual deterioration, not before a few, up to ten, years. This is confirmed by literature data [1-3,8].

The vast majority of our patients presented the classical form of PMD but of variable clinical severity even within the same family and the same mutation. This observation together with the small number of patients means that we cannot conclude about any genotype-phenotype correlations. Moreover, the patient with the connatal form of PMD also carried the duplication of the entire gene just as the patients with the classic form, which additionally confirmed the absence of such a correlation.

Differences in the clinical picture were rather related to the time when certain signs appeared as well as their intensity rather than their type. They were neither characteristic nor significant, so they did not result in any new elements in the diagnostic criteria. The variability of the disease course observed in siblings was also seen by other authors $[1-3,8]$. It can be explained by influence of the remaining genetic material, as well as epigenetic and environmental factors $[2,8]$.

The diagnosis in all probands but one, just as in other rare genetic disorders, was established late, after a few up to over ten years after the appearance of the first symptoms of the disease $[2,7]$. Due to its $X$-linked inheritance, the diagnosis of PMD in familial cases may be enhanced by pedigree analysis if males are affected along the feminine line of inheritance. A proportion of PMD cases may develop de novo, which is not always acknowledged by specialists other than geneticists.

The detection of hypomyelination of the central nervous system is an indispensable element to suspect PMD or other hypomyelinating leukodystrophy (HLD). It can be demonstrated exclusively by magnetic resonance imaging (MRI) with analysis of individual time sequences, particularly at T2 and FLAIR [3]. Unfortunately, some of the probands initially had only computed tomography (CT) performed, most frequently for economic reasons. CT does not allow for correct differentiation of the lesion type (hypo-, dys- or demyelination).

Brain auditory evoked potentials (BAEP) are a second medical test which may detect hypomye- 
lination but only differentiates this type of abnormality from the other white matter lesions. So the examination is not helpful and additionally available in just a few diagnostic centers.

Fundoscopic examination showed in PMD patients small, hypoplastic optic discs or their pallor, related to hypoplasia or atrophy, but not characteristic of the disease. It can be detected in several inborn or acquired diseases of the white matter [11]. It should be underlined that despite this lesion patients preserve vision for a long time.

The only investigation that can confirm the diagnosis of PMD is molecular analysis, demonstrating mutations in the PLP1 gene [2,8]. In our material molecular confirmation of PMD was obtained in 15 cases per 68 DNA samples (around 20\%) submitted for testing. Duplications encompassing 7 exons (the entire gene) were found in 7 cases, whereas point mutations of missense or nonsense type (see also the Table) were demonstrated in the remaining cases $[4,5]$. The duplication results in overproduction of PLP and DM20 proteins while the remaining mutations result in their abnormal (missense) or truncated (nonsense) form $[2,8]$.

In the remaining 39 patients mutations have not been detected. It might be due to still insufficient molecular investigative techniques or to the fact that a similar clinical picture may result from other genetic causes. For the last two decades molecular genetics development have allowed to demonstrate heterogeneity of HLD $[8,11,12]$. Apart from PMD, more than ten HLD were established, manifesting various types of the inheritance pattern $[6,8,11]$. The clinical course of these diseases may be similar to that of PMD or sometimes additional signs are present e.g. hypodontia, cataract or atrophy of basal ganglia on MRI [9-12]. Moreover, world experts in the white matter diseases state that unclassified leukodystrophies with hypomyelination still exist $[9,11]$. These new findings may be accessed by available online international internet data bases, such as OMIM or www.genereview. org, that are regularly updated.

\section{Conclusions}

The classical PMD form represents a slowly progressing leukodystrophy, with apparent clinical improvement in the first years and deterioration not observed until the later period of life. The MRI is an essential method for detection and differentiation of white matter lesion types. The diagnosis of PMD can be confirmed only with molecular analysis. Genetic confirmation of the diagnosis allows to cover the family with genetic counselling and to suggest an appropriate supportive therapy. It also terminates a longstanding and very expensive diagnostic process, which in addition can be emotionally very painful for all the family members.

It is highly probable that a considerable number of PMD patients remain undiagnosed, so boys with a "label" of cerebral palsy should be watched carefully for alternative diagnosis.

\section{Acknowledgements}

We are sincerely grateful to our patients and their parents for their participation in the study as well for their informed consent to DNA analysis and publication. We are also grateful to doctors Antoni Kierdaszuk, Jolanta Wierzba and Anna Jakubiuk-Tomaszuk who referred the patients to our center.

\section{Disclosure}

Authors report no conflict of interest.

\section{References}

1. Cailloux F, Gauthier-Barichard F, Mimault C, Isabelle V, Courtois V, Giraud G, Dastugue B, Boespflug-Tanguy O. Genotype-phenotype correlation in inherited brain myelination defects due to proteolipid protein gene mutations. Eur J Hum Genet 2000; 8: 837-845.

2. Garbern JY, Krajewski K, Hobson G. PLP1-related disorders. Gene Reviews 2010; available at: http://www.ncbi.nlm.nih. gov/books/NBK1182 [Accessed February 2015].

3. Grossi S, Regis S, Biancheri R, Mort M, Lualdi S, Bertini E, Uziel G, Boespflug-Tanguy O, Simonati A, Corsolini F, Demir E, Marchiani V, Percesepe A, Stanzial F, Rossi A, Vaurs-Barrière C, Cooper DN, Filocamo M. Molecular genetic analysis of the PLP1 gene in 38 families with PLP1-related disorders: identification and functional characterization of 11 novel PLP1 mutations. Orphanet J Rare Dis 2011; 6: 40.

4. Hoffman-Zacharska D, Mierzewska H, Szczepanik E, Poznański J, Mazurczak T, Jakubiuk-Tomaszuk A, Mądry J, Kierdaszuk A, Bal J. The spectrum of PLP1 gene mutations in patients with the classical form of the Pelizaeus-Merzbacher disease. Med Wieku Rozwoj 2013; 17: 293-300.

5. Hoffman-Zacharska D, Mierzewska H, Mazurczak T, Derwińska K, Górka K, Kłyszejko J, Szkudlińska-Pawlak S, Szczepanik E. Connatal Pelizaeus-Merzbacher disease caused by Xq21-22 submicroscopic duplication including the PLP1 gene - case report. Neurol Dziec 2013; 45: 73-77 [in Polish]. 
6. Lyons G, Kolodny EH, Pastores GM. Neurology of hereditary metabolic disorders of children. $3^{\text {rd }}$ ed. McGraw-Hill, New York 2006.

7. Mądry J, Hoffman-Zacharska D, Królicki L, Jakuciński M, Friedman A. PLP1 gene duplication as a cause of the classic form of Pelizaeus-Merzbacher disease - case report. Neurol Neurochir Pol 2010; 44: 511-515.

8. OMIM. Available online at www.ncbi.nlm.nih.gov/omim [Accessed February 2015].

9. Schiffmann R, van der Knaap MS. Invited Article: an MRI-based approach to the diagnosis of white matter disorders. Neurology 2009; 72: 750-759.

10. Steenweg ME, Vanderver A, Blaser S, Bizzi A, de Koning TJ, Mancini GM, van Wieringen WN, Barkhof F, Wolf NI, van der Knaap MS. Magnetic resonance imaging pattern recognition in hypomyelinating disorders. Brain 2010; 133: 2971-2982.

11. Vanderver A, Tonduti D, Schiffmann R, Schmidt J, Van der Knaap MS. Leukodystrophy overview. In: Pagon RA, Adam MP, Ardinger HH, Bird TD, Dolan CR, Fong CT, Smith RJH, Stephens K (eds.). GeneReviews ${ }^{\circledR}$ [Internet]. Seattle (WA): University of Washington, Seattle; 1993-2014. http://www.ncbi.nlm.nih.gov/ books [Accessed February 2015].

12. Wolf NI, Vanderver A, van Spaendonk RM, Schiffmann R, Brais B, Bugiani M, Sistermans E, Catsman-Berrevoets C, Kros JM, Pinto PS, Pohl D, Tirupathi S, Strømme P, de Grauw T, Fribourg S, Demos M, Pizzino A, Naidu S, Guerrero K, van der Knaap MS, Bernard G; 4H Research Group. Clinical spectrum of 4H leukodystrophy caused by POLR3A and POLR3B mutations. Neurology 2014; 83: 1898-1905. 